\title{
EL IMPACTO DE LAS VARIABLES MACROECONOMICAS EN LA RENTABILIDAD DE LA BOLSA DE VALORES DE LIMA
}

\author{
THE IMPACT OF THE MACROECONOMIC VARIABLES ON THE PROFITABILITY OF \\ THE LIMA STOCK EXCHANGE
}

Pedro Pablo Chambi Condori Universidad Nacional Jorge Basadre Grohmann Tacna, Perú

ORCID: https://orcid.org/0000-0002-2565-2780

Correo electrónico: pchambic@unjbg.edu.pe

\section{RESUMEN}

Objetivo: El objetivo principal de la investigación está orientado a explicar las implicancias que ejercen las variables macroeconómicas sobre la rentabilidad de la Bolsa de Valores de Lima. Método: La metodología a seguir consiste en la utilización del modelo de mínimos cuadrados para la expresión de un modelo multivariante con data histórica de las variables macroeconómicas del Perú, tales como la tasa de crecimiento del PBI (CE), el tipo de cambio (TC), la tasa de interés (TI) la inflación (IP) y la variable dependiente: retornos de la Bolsa de Valores de Lima (R_IGBVL). Resultados: Obtenido el modelo econométrico, se confirma la incidencia positiva de las variables tasa de crecimiento del PBI y tipo de cambio sobre los retornos de la Bolsa de Valores de Lima. Por otro lado, efectos negativos por parte de las variables tipo de interés e inflación sobre los retornos de la Bolsa de Valores de Lima. Conclusiones: En concordancia con la teoría de análisis fundamental top down de mercados financieros, se evidencia la incidencia positiva de las variables crecimiento de la economía peruana y tipo de cambio sobre los retornos de la Bolsa de Valores de Lima, en razón de la presencia fortalecida de acciones mineras en el mercado bursátil peruano. Por otro lado, una relación negativa por parte de las variables tipo de interés y tasa de inflación.

Palabras clave: Mercado de capitales; rentabilidad; variables macroeconómicas; retornos.

\begin{abstract}
Objective: The main objective of the research is aimed to explain the implications of macroeconomic variables on the profitability of the Lima Stock Exchange. Method: The -methodology to follow is the use of the least- squares model for the expression of a multivariate model with historical data from Peru's macroeconomic variables, such as the growth rate of GDP (CE), exchange rate (TC), interest rate (IT), inflation (IP), and the dependent variable: returns of the Lima Stock Exchange (R_IGBVL). Results: Obtained the econometric model, confirms the positive impact of GDP growth rate and exchange rate variables on the returns of the Lima Stock Exchange. Conclusions: In line with the top down fundamental analysis theory of financial markets, it evidences the positive incidence of the variables growth Peruvian economy and exchange rate on the returns of the Lima Stock Exchange, by reason of the strengthened presence of mining stocks on the Peruvian stock market. One the other hand, a negative relationship on the part of the interest rate and inflation rate variables.
\end{abstract}

Keywords: Capital market; profitability; Macroeconomic variables; returns.

(c) Los autores. Este artículo es publicado por la Revista Quipukamayoc, Universidad Nacional Mayor de San Marcos. Este es un artículo de acceso abierto, distribuido bajo los términos de la Licencia Creative Commons Atribución-NoComercial-Compartirlgual 4.0 Internacional.(http://creativecommons.org/licenses/by-nc-sa/4.0/), que permite el uso no comercial, distribución y reproducción en cualquier medio, siempre que la obra original sea debidamente citadas. 


\section{INTRODUCCIÓN}

El mercado de capitales es un mercado en el que se negocian las acciones de empresas públicas y privadas, en el que opera el mecanismo de transferencia de fondos desde los superavitarios hacia los deficitarios. Constituye un mercado en donde los agentes deficitarios encuentran financiamiento, mientras que los superavitarios estructuran sus carteras de inversión en activos financieros; asimismo, a través del mercado secundario otorga liquidez para los tenedores de títulos y valores.

La asociación entre las variables macroeconómicas y el performance alcanzado en los mercados bursátiles ha sido ampliamente estudiado y documentado en los mercados de capitales desarrollados, tales como los de Estados Unidos, Japón, Australia y Canadá, y en los mercados europeos de España, Alemania y Francia (Chen, Roll y Ross, 1986).

La salud de la economía puede afectar al desempeño del mercado de valores de varias maneras. Las fuerzas económicas afectan a las tasas de descuento, la capacidad de las empresas para generar flujos de efectivo y futuros pagos de dividendos. Es a través de este mecanismo que las variables macroeconómicas se convierten en parte de los factores de riesgo en el mercado de valores. Una mayor demanda de fondos para préstamos da como resultado un aumento en la tasa de interés real, lo que reduce el valor presente de los flujos de efectivo futuros de una empresa y hace que caigan los precios de las acciones. Los modelos orientados al flujo plantean una asociación positiva entre la producción y los precios de las acciones a través del tipo de cambio (Mishkin, 2008, p. 134).

Bahloul, Mroua y Naifar (2016), han investigado el impacto de las variables macroeconómicas en la rentabilidad del índice islámico, encontrando incidencia indirecta por las variables del tipo de interés y la tasa de inflación, y por otro lado impactos positivos por parte de las variables de crecimiento de la economía y del incremento en el flujo de las inversiones indirectas.

Balagobei (2017), ha estudiado la relación que existe entre las variables macroeconómicas y la rentabilidad de la bolsa de valores de Sri Lanka, encontrando impactos positivos en la rentabilidad bursátil por parte de las variables de crecimiento de la economía de Sri Lanka y el tipo de cambio, y relación indirecta con las variables de la tasa de inflación y la tasa de interés, estudio realizado en el periodo 2006-2015.

Abdul (2008), ha realizado la investigación sobre la relación dinámica que existe entre las variables macroeconómicas y la rentabilidad de la bolsa de valores de Pakistán en el periodo de 2001 a 2007 utilizando el modelo de cointegración, encontrando el impacto positivo por parte del crecimiento de la economía en la rentabilidad del mercado bursátil de Pakistán y, por otro lado, la relación indirecta con la tasa de inflación.

Pájaro y Ramos (2015), han investigado la relación que se presenta entre las variables macroeconómicas y el índice general de la Bolsa de Valores de Colombia, en el periodo comprendido entre 2003 a 2012, encontrando una relación positiva entre las variables de crecimiento de la economía y una relación indirecta con la tasa de interés interna y el tipo de cambio nominal.

Morales y Aguilera (2018) refieren que en una economía en crecimiento, los componentes de la demanda interna agregada como el gasto de consumo, la inversión y el gasto público tienden a expandirse, propiciando que las empresas expresen incremento en sus ventas y consecuentemente obtengan mayores utilidades, y por tanto, incrementos en $R O E=\left(\frac{\text { Utilidad neta }}{\text { Patrimonio }}\right)$ y mejores indicadores de $P E R=\left(\frac{\text { Precio de mercado }}{\text { Utilidad }}\right)$, en donde:

ROE: Rentabilidad del patrimonio.

$\mathrm{PER}=$ precio/utilidad, indicador que depende de la gestión económica de la organización y por otro lado de la valoración de las acciones que tengan los inversionistas en el mercado de acciones, por lo que este indicador está íntimamente relacionado con la política de dividendos que imprima una organización en la gestión de la entrega de dividendos a sus accionistas.

ROE es muy importante para los tenedores de acciones, porque tiene una relación directa con los dividendos que reciben los accionistas, esta relación esta expresada en la ecuación siguiente:

$$
\text { Dividendos }=\text { Pay out } *\left[\frac{\text { Utilidad neta }}{\text { Acción }}\right]
$$

Pay out: Porcentaje de entrega de utilidad como dividendos. Depende de la política de dividendos que gestiona la organización. En este apartado, es preciso puntualizar que una acción restrictiva de la política de dividendos repercute negativamente en el valor del PER, por efecto de la disminución del precio de las acciones en el mercado bursátil, y una acción expansiva en la política de dividendos tiene implicancia positiva en el precio de las acciones que se negocian en el mercado bursátil.

$\frac{\text { Utilidad neta }}{\text { Acción }}$ : Utilidad por cada acción, expresado en unidades monetarias.

El efecto financiero de la tasa de interés sobre el precio de las acciones es negativo, dado que en la medida que se incrementa la tasa de interés es beneficiosa para el mercado 
de renta fija, pero el efecto sobre el precio de las acciones es negativo. Por consiguiente, el efecto sustitución entre los instrumentos de renta fija y de renta variable se puede considerar como un efecto de corto plazo como consecuencia del movimiento de las tasas de interés pasivas sobre la tendencia del precio de las acciones en el mercado secundario de valores. La inflación significa pérdida del poder adquisitivo, y provoca un efecto negativo sobre el consumo privado, consecuentemente tiene efectos negativos en el precio de las acciones. El efecto financiero del tipo de cambio sobre el precio de las acciones es adverso, debido a la depreciación de la moneda nacional en corto plazo ejerce un efecto de contracción del precio de las acciones, pero para el caso de las empresas exportadoras el impacto es beneficioso debido al incremento de competitividad de los bienes y servicios de origen nacional en el mercado internacional, por lo tanto, las empresas obtienen mayores ingresos por ventas y rentabilidad, lo que fortalece su situación financiera.

El objetivo de la investigación es explicar el comportamiento del desempeño del mercado bursátil peruano en relación al impacto de las variables macroeconómicas del país en el período del 2000 al 2019.

El análisis fundamentalista top down de un mercado bursátil centra su estudio en examinar el comportamiento del PBI de una economía. Si esta variable manifiesta un crecimiento sostenido en el tiempo su efecto en el mercado bursátil de renta variable es positiva, por otro lado, una tasa de inflación alta y consecuentemente una tasa de interés también alta tienen incidencia negativa sobre la rentabilidad del mercado accionario y positiva para el mercado de dinero; y a la inversa, si el de interés es baja y también baja la tasa de inflación, su efecto es favorable para el mercado de acciones y negativa para el mercado de bonos (Martín, 2011, p. 80).

Performance del mercado de acciones $=f($ variables fun damentales top down)

Que para el tema de investigación el modelo teórico se traduce en la expresión siguiente:

$R_{-} I G B V L_{t}=f(C E, T C, T I, I P)$, en donde $R_{-} I G B V L_{t}$ es la serie de tiempo de los retornos de la Bolsa de Valores de Lima, CE es la serie de tiempo del crecimiento de la economía peruana, TC es el tipo de cambio de la relación de la moneda nacional con la divisa norteamericana, TI es la tasa de interés interna, IP es la serie de tiempo de la tasa de inflación de la economía peruana.

La hipótesis formulada para la presente investigación consiste en explicar la evidencia del impacto de las variables macroeconómicas en los retornos de la Bolsa de Valores de Lima.
Desde esta perspectiva, conocer el impacto de cada una de las variables es muy importante, tanto para los inversionistas en la selección de los mercados financieros de inversión a tener en cuenta en la selección de los destinos de inversión, como para la estructuración del portafolio de inversiones.

\section{MATERIALES Y MÉTODOS}

En el contexto macroeconómico peruano, para el desarrollo de la investigación se ha dispuesto de los datos que se describen a continuación:

- Los datos diarios del índice general de la Bolsa de Valores de Lima (BVL) desde el 3 de enero del 2000 hasta el 31 de diciembre del año 2019, obtenidos a partir de la base de datos de la BVL.

- Con el fin de expresar en términos de rentabilidad la data del índice general de la Bolsa de Valores del Perú se ha utilizado la ecuación siguiente:

- $\quad \operatorname{Rent}_{B V L}=100 * \ln \left(\frac{P_{1}}{P_{0}}\right)$, en donde $P_{0}$ es el valor del IGBVL del día anterior y $P_{1}$ es el valor del IGBVL del día, en términos porcentuales.

- Los datos del crecimiento de la economía peruana desde el año 2000 hasta el año 2019, representado en este caso por el PBI anual en términos porcentuales.

- Los datos del tipo de cambio promedio anual desde el año 2000 hasta el año 2019, del comportamiento de la moneda nacional respecto a la divisa estadounidense.

- Los datos de la tasa de interés de referencia establecida por el Banco Central de Reserva del Perú (BCRP), luego expresado en promedio anual.

- Los datos del índice de precios, expresado en términos porcentuales y promedio anual.

Una vez obtenidos los datos históricos, estos fueron procesados para obtener el modelo de análisis multivariante de la forma que expresa la ecuación siguiente:

$R_{-} I G B V L_{t}=\beta_{0}+\beta_{1} C E_{t}+\beta_{2} T C_{t}+\beta_{3} I P_{t}+\beta_{4} T I_{t}+\varepsilon_{t^{\prime}}$ en donde:

$R_{-} I G B V L_{t}:$ Rentabilidad de la Bolsa de Valores de Lima, expresado en porcentaje.

$C E_{t}$ : Crecimiento de la economía peruana.

$P_{t}:$ Índice de precios al consumidor.

$T C_{t}$ :Tipo de cambio de soles respecto al dólar estadounidense.

$T_{t}$ : Tipo de interés de referencia bancaria. 
$B_{0}$ : Valor del intercepto.

$B_{1}, B_{2}, B_{3}, B_{4}$ : Los coeficientes de cada una de las variables incidentes.

\section{RESULTADOS}

En una economía, el mercado de capitales tiene una singular importancia; además, la mayoría de las economías en crecimiento están dotadas de mercados bursátiles en crecimiento, como es el caso del mercado bursátil peruano.

Tal como se puede observar en la figura 1, el desempeño de la Bolsa de Valores de Lima en el periodo del 2002 al 2007 ha sido una de las más importantes en los mercados emergentes, y según el BCRP es el periodo en el que se manifiesta un crecimiento sostenido de la economía peruana, y que después, del 2008 al 2019 ha mostrado comportamientos de desempeño dentro de márgenes de alta volatilidad.

En la imagen superior de la figura 1, se aprecia los cambios en el crecimiento económico expresado en millones de dólares, mientras que en la parte posterior se puede expresar en términos porcentuales la volatilidad de la economía peruana en el período 2000 al 2019.

Los datos ya organizados en el periodo 2000-2019 de las variables del modelo en la investigación, se exponen en la tabla 1.

\section{IGBVL}
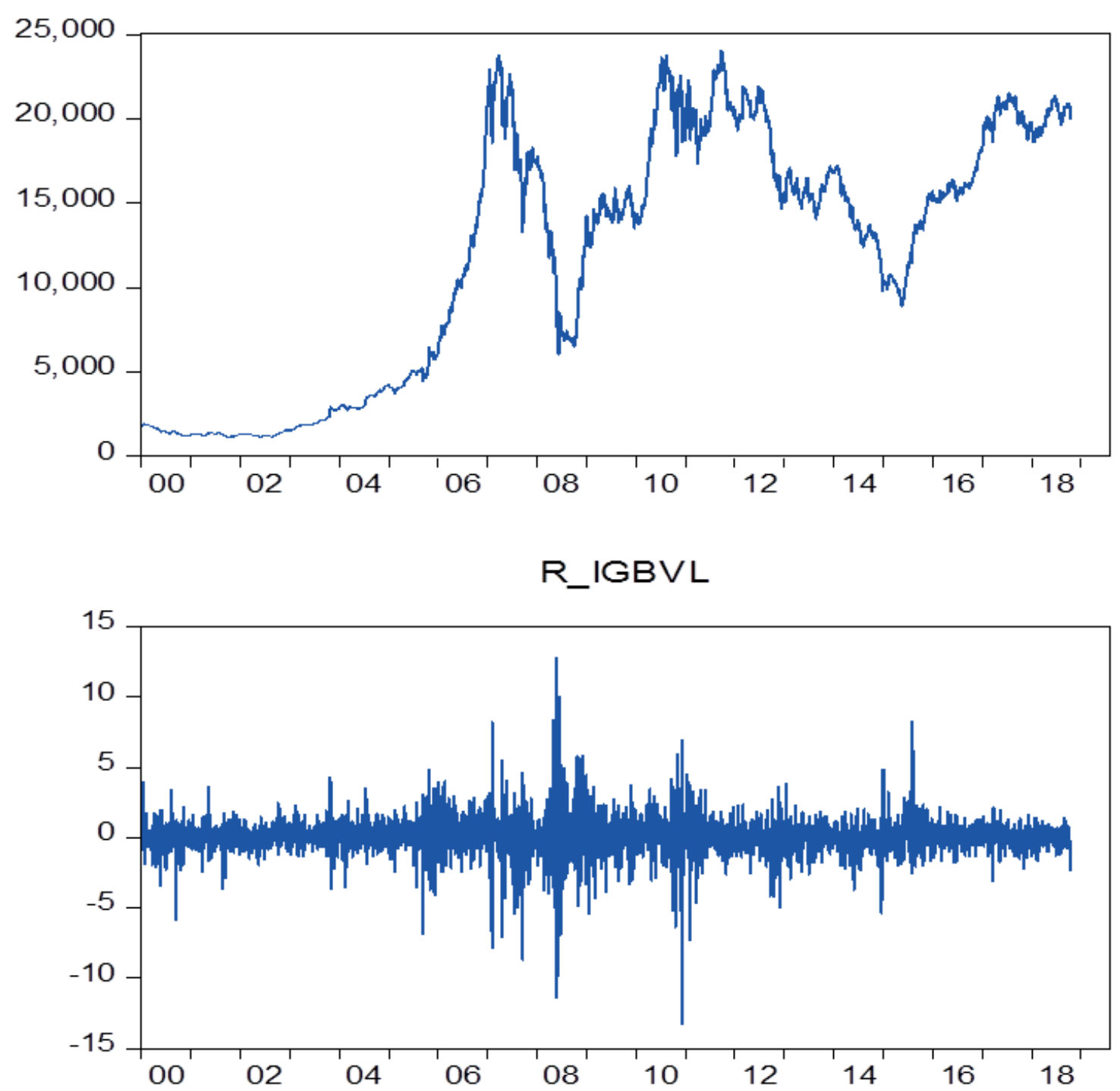

Figura 1. Evolución histórica del IGBVL y los retornos de la Bolsa peruana (2000-2019). 
Tabla 1

Las variables macroeconómicas y la rentabilidad de la Bolsa de Valores de Lima

\begin{tabular}{cccccc}
\hline AÑO & TC & CE\% & IP\% & TI\% & R_IGBVL\% \\
\hline 2000 & 3,430 & 2,700 & 3,750 & 3,800 & $-0,1663$ \\
2001 & 3,530 & 0,600 & 1,970 & 3,100 & $-0,0108$ \\
2002 & 3,518 & 5,500 & 1,530 & 3,800 & 0,0687 \\
2003 & 3,479 & 4,200 & 2,260 & 2,750 & 0,2237 \\
2004 & 3,414 & 5,000 & 3,660 & 3,000 & 0,1685 \\
2005 & 3,297 & 6,300 & 1,610 & 3,250 & 0,1028 \\
2006 & 3,275 & 7,500 & 2,000 & 4,500 & 0,3932 \\
2007 & 3,129 & 8,500 & 1,780 & 5,000 & 0,1230 \\
2008 & 2,926 & 9,100 & 5,790 & 1,500 & $-0,3672$ \\
2009 & 3,012 & 1,100 & 2,940 & 3,000 & 0,2804 \\
2010 & 2,826 & 8,300 & 1,520 & 4,250 & 0,1995 \\
2011 & 2,755 & 6,300 & 3,360 & 4,250 & $-0,0725$ \\
2012 & 2,638 & 6,100 & 2,650 & 4,000 & 0,0230 \\
2013 & 2,703 & 5,900 & 3,080 & 3,500 & $-0,1070$ \\
2014 & 2,839 & 2,400 & 3,200 & $-0,0249$ \\
2015 & 3,186 & 3,300 & 4,130 & 3,750 & $-0,1634$ \\
2016 & 3,377 & 3,900 & 3,340 & 1,700 & 2,250 \\
2017 & 3,262 & 3,500 & 2,400 & 3,250 & 0,1831 \\
2018 & 3,288 & 2,300 & 1,880 & 2,750 & 0,0993 \\
2019 & 3,339 & & 2,250 & $-0,0126$ \\
\hline
\end{tabular}

Fuente: Banco Central de Reserva del Perú y Bolsa de Valores de Lima.

Los resultados descriptivos de cada una de las variables que se analizan en el modelo se exponen en los gráficos de la figura 2, en los que se aprecia el comportamiento que ha experimentado cada una de las variables en el horizonte estudiado.

En la figura 2, se puede apreciar una correspondencia en el movimiento temporal entre el CE y la rentabilidad de la Bolsa de Valores de Lima (RENT_IGBVL) y de manera similar con el tipo de interés (TI) y el tipo de cambio (TC).

Procurado el procesamiento estadístico de datos con la data histórica presentada en la tabla 1 , se tiene el resultado de indicadores estadísticos que se presenta en la tabla 2.

Con la información obtenida y presentada en la tabla 2, se expresa el modelo de los retornos de la Bolsa de Valores de Lima, en la forma que se expone a continuación:

Modelo (1):

$R_{-} I G B V L_{t}=0.0239+0.0315 * C E_{t}+0.1121 * T C_{t}-0.0873$

* $\mathrm{TI}_{t}-0.0605 * I P_{t}+\varepsilon_{t}$
De la lectura de los coeficientes del modelo se puede visualizar que la variable que tiene impacto de primer nivel en los retornos de la Bolsa de Valores de Lima es el tipo de cambio (TC) con el valor en el coeficiente de 0,1121 ; el de segundo lugar en importancia es el crecimiento de la economía peruana, expresada para el caso por la variación del PBI, con un coeficiente de 0,0315; sin embargo, la variable tasa de interés (TI) y la inflación (IP) tienen implicancia negativa. El estadístico DW=2,2856 está dentro del rango aceptado, por tanto, se puede afirmar que no existe evidencia de autocorrelación entre las variables que explican el modelo y por el valor del coeficiente de determinación de 0,53 se concluye que el $47 \%$ del comportamiento de la rentabilidad de la Bolsa de Valores de Lima, estadísticamente está explicada por otras variables que no son las macroeconómicas. En ese mismo sentido tiene la interpretación de los estadísticos F, T y la probabilidad a 95\% de nivel de confianza.

Con los resultados ya expresado en el modelo (1), se puede afirmar que las variables macroeconómicas sí tienen incidencia en el rendimiento de la Bolsa de Valores de Lima, confirmando con ello la teoría del análisis fundamental top 
CE

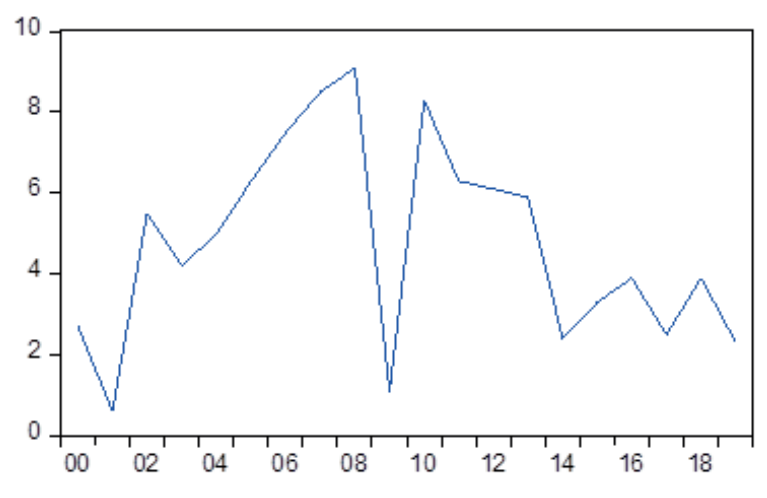

RENT_IGBVL

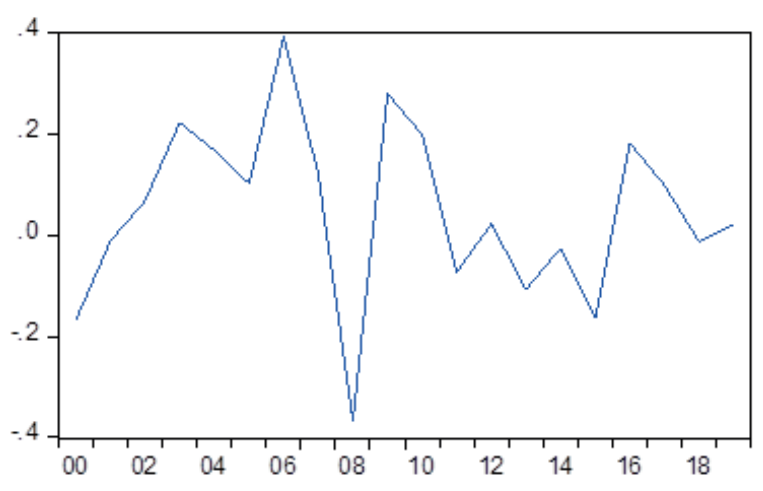

$\mathrm{Tl}$

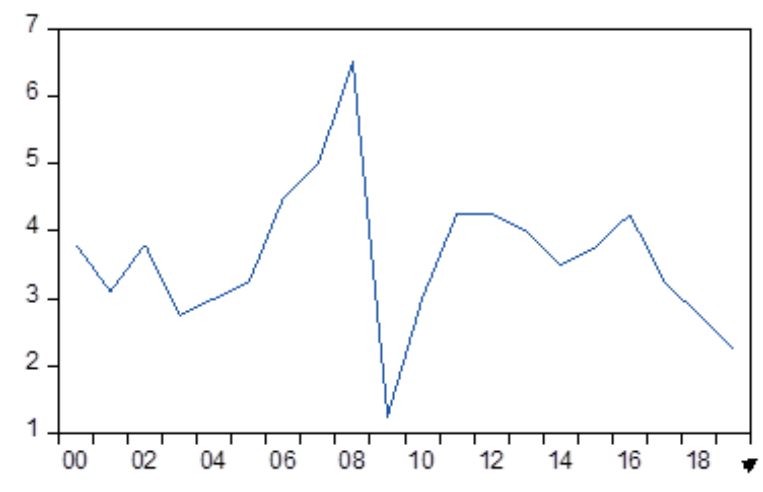

IP

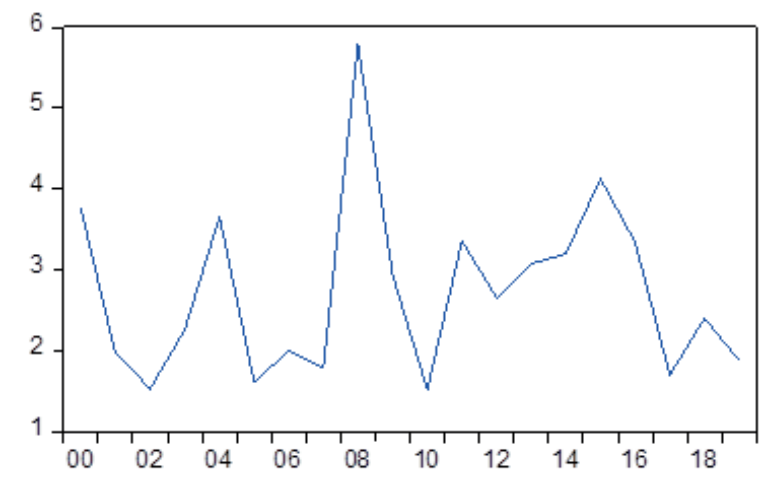

TC

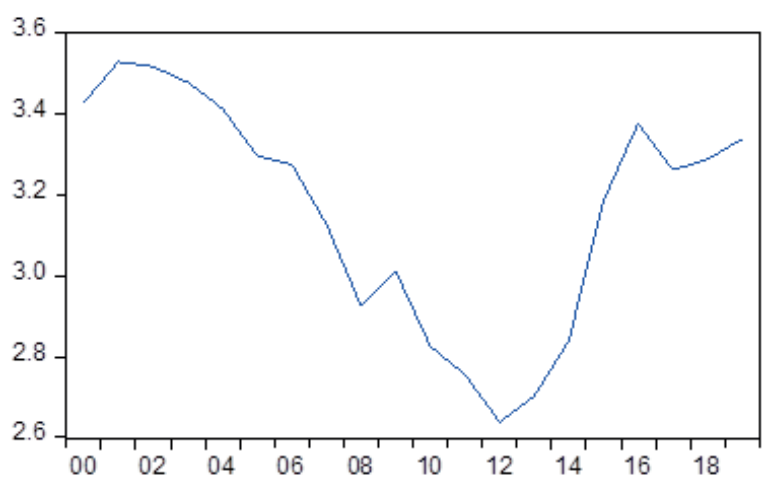

Figura 2. Serie de tiempo de las variables que explican el modelo (2000-2019)

down (Martin, 2011), incidencia positiva por parte del crecimiento de la economía (CE) y del tipo de cambio (TC); y la incidencia negativa por parte de la tasa de interés (TI) y de la inflación (IP), con lo que queda demostrado el supuesto de la investigación.

\section{DISCUSIÓN}

De acuerdo a los resultados obtenidos en la investigación y presentados en la tabla 2, se confirma que las variables que tiene efectos favorables para el mercado bursátil peruano son el crecimiento económica y el tipo de cambio, que según la teoría de análisis fundamental top down de Martín (2011), el crecimiento sostenido de una economía tiene impacto favorable en el mercado de acciones; y por otro lado, la incidencia de las variables de inflación (IP) y la tasa de interés (TI) es negativa sobre el mercado de acciones; $y$, efectivamente, en el presente estudio se corroboran las afirmaciones de Martín, tal cual se puede apreciar en el modelo (1). En 
Tabla 2

Modelo de rentabilidad de la Bolsa de Valores de Lima

\begin{tabular}{|c|c|c|c|c|}
\hline \multicolumn{5}{|c|}{ Dependent Variable: RENT_IGBVL } \\
\hline \multicolumn{5}{|l|}{ Method:Least quare } \\
\hline \multicolumn{5}{|c|}{ Date: $02 / 25 / 20$ Time:22:33 } \\
\hline \multicolumn{5}{|l|}{ Sample:2000 2019} \\
\hline \multicolumn{5}{|c|}{ Included observations:20 } \\
\hline Variable & Coefficient & Std. Error & t-Statistic & Prob. \\
\hline C & 0,0239 & 0,4392 & 0,0545 & 0,9573 \\
\hline CE & 0,0315 & 0,0199 & 15,827 & 0,1343 \\
\hline $\mathrm{TC}$ & 0,1121 & 0,1227 & 0,9130 & 0,3757 \\
\hline $\mathrm{TI}$ & 0,0873 & 0,0476 & 18,331 & 0,0867 \\
\hline IP & 0,0605 & 0,0363 & 16,691 & 0,1158 \\
\hline R-squared & 0,5304 & \multicolumn{2}{|c|}{ Mean dependent var } & 0,0481 \\
\hline Adjusted R-squared & 0,4052 & \multicolumn{2}{|c|}{ S.D.dependent var } & 0,1757 \\
\hline S.E. of regression & 0,1355 & \multicolumn{2}{|c|}{ Akaike info criteriom } & 0,9468 \\
\hline Sum squared resid & 0,2756 & \multicolumn{2}{|c|}{ Schwarz criterion } & 0,6979 \\
\hline Log likelihood & 144,683 & \multicolumn{2}{|c|}{ Hannan-Quinn criter } & 0,8982 \\
\hline F-statistic & 42,358 & \multicolumn{2}{|c|}{ Durbin-Watson stat } & 22,856 \\
\hline Prob(F-statistic) & 0,0172 & & & \\
\hline
\end{tabular}

Fuente: BCRP y la Bolsa de Valores de Lima.

el modelo (1), el coeficiente positivo del tipo de cambio (TC) está explicado por la fortaleza que tienen las acciones mineras que se negocian en el mercado bursátil peruano. Los resultados obtenidos por Balagobei (2017) en Sri Lanka, ha encontrado que las variables de crecimiento económico y el tipo de cambio tienen efectos positivos en el mercado accionario, y por otro lado, la tasa de interés y la inflación tienen efectos negativos. Por otro lado, el estudio de Pájaro y Castro (2015) en Colombia, han encontrado resultados de incidencia favorable en el índice general de la Bolsa de Valores de Colombia por parte del crecimiento de la economía y una incidencia negativa por parte de las variables de tasa de interés interna y el tipo de cambio; en esta última variable de tipo de cambio difiere con nuestros resultados obtenidos, esto en razón del componente de acciones mineras en el mercado bursátil peruano.

\section{REFERENCIAS}

Aguilera, G. y Morales, A. (2018). Contexto Macroeconómico y Precio de las Acciones. México DF: Ediciones ECOE.

Bahloul, S., Mroua, M. y Naifar, N. (2016). The impacto of macroecnomic and convencional sotck market variables on Islamic index returns under regime switching. Borsa Istanbul Review 17-1(2017)62-74
Balagobei, S. (2017). Macroeconomic variables and Stock Market Returns in Sri Lanka. Asian Journal of Finance \& Accounting, ISSN 1946-052X, 2017, Vol 9, №.2. University of Jaffna.

Chen, N., Roll, R. y Ross, S. (1986). Economic forces and the stock markets. Journal of Business 59, 383-403, https://doi.org/10.1086/296344

Martín, M. (2011). Mercado de Capitales: una perspectiva global. Buenos Aires: editorial Cengage Learning.

Mishkin, F. (2008). Moneda, Banca y Mercados Financieros. México D.F.: Editorial Pearson.

Pájaro, A. y Ramos, G. (2015). Determinantes macroeconómicos del comportamiento del índice general de la Bolsa de Valores de Colombia. Cartagena: Universidad de Cartagena.

Rashid, A. (2008). Macroecnomic Variables and Stock Market Performance: Testing for Dynamic Linkages with Known Structural Break. International Islamic University, Pakistan. 
